Classification

Physics Abstracts

$61.25 \mathrm{H}-87.10$

\title{
Kinetics of collapse for a flexible coil
}

\author{
P. G. de Gennes \\ Collège de France, 75231 Paris Cedex 05, France
}

(Reçu le 3 avril 1985, accepté le 20 mai 1985)

\begin{abstract}
Résumé. - Si on transfère tout à coup une chaîne flexible depuis le point $\theta$ vers une situation de mauvais solvant (par exemple, par un changement de température $\Delta T$ ) elle s'effrondre en un temps $\tau_{\mathrm{c}}$. Nous proposons ici une loi d'échelle pour $\tau_{\mathrm{c}}$, basée sur un modèle de « saucisse gonflée ", et prenant en compte les interactions hydrodynamiques. Nous trouvons $\tau_{c} \cong \tau_{R} \Delta T / \theta$ où $\tau_{R}$ est le temps de Rouse de la chaîne au point $\theta$ : le temps $\tau_{\mathrm{c}}$ croît avec la profondeur de la trempe.

Abstract. - If a polymer chain is transferred abruptly from $\theta$ conditions to bad solvent conditions (e.g. by a shift of temperature $\Delta T$ ) it collapses with a certain characteristic time $\tau_{\mathrm{c}}$. We propose a scaling structure for $\tau_{c}$, based on an "expanding sausage model ", and taking into account the hydrodynamic interactions. We find that $\tau_{c} \cong \tau_{\mathrm{R}} \Delta T / \theta$ where $\tau_{\mathrm{R}}$ is the Rouse time of the $\theta$ chain : the time $\tau_{\mathrm{c}}$ increases with the depth of quench.
\end{abstract}

\section{Aims and tools.}

Proteins are synthetized as flexible polypeptides; they collapse into a specific, compact form. The kinetics of this folding process are amazingly complex [1]. This is largely due to the chemical variability of the aminoacid sequence. In earlier work, we have attempted to analyse the slowing down of certain reptation motions, in the compact phase, due to the chemical heterogeneity [2]. In the present paper, our aim is even more modest : we restrict our attention to a linear homopolymer of $N$ monomers, each of size $a$. We start from $\theta$ conditions, and drop the temperature by an amount $\Delta T$. We must have $\Delta T>\theta / N^{1 / 2}$ to reach a significant collapse [3]. The final state has been widely studied experimentally [4-8] and theoretically - starting from an idea of Stockmayer [9]. Here we shall use the simplest (mean field) theoretical description [10, 11] to describe the collapse.

We may divide the chain into «blobs " of $g=(\theta / \Delta T)^{2}$ monomers and of size $\xi=a g^{1 / 2}$. Inside one subunit the shift $\theta \rightarrow \theta-\Delta T$ has negligible effects. But adjacent subunits have an attractive potential of order $(-k \theta)$. The final state is a dense piling of subunits, with a concentration $c_{a} \sim g / \xi^{3}$.

How do we reach this final state ? A first - tempting but incorrect - approach amounts to replace the collapsing coil by a sphere of radius $R(t)$ and uniform concentration $\bar{c}=N R^{-3}$; then to estimate the mean field interaction energy associated with $\bar{c}$; and finally to equate the time derivative of this energy to the viscous losses. This is wrong, however, because the coil is very far (initially) from a homogeneous sphere. It is preferable to think of the initial state as a string of $N / g$ blobs, and to assume that the blobs tend to stick together, so as to minimize the 
surface energy between an inner domain of concentration $c_{g}$ and free solvent. The interfacial energy is [10] :

$$
\gamma \sim k T / \xi^{2} \sim k \theta / \xi^{2}
$$

The shape of the inner domain is not a sphere, but is probably close to a " sausage " of length $L(t)$ and radius $r(t)$ (Fig. 1).

At the starting point $(t=0)$ we have $L=L_{0}=N / g \xi$ and $r=\xi$. At later times the sausage swells laterally and contracts longitudinally, keeping a constant volume $\Omega=\pi r^{2} L$. We focus our attention on this sausage model, and assume that the sausage may be treated as rectilinear (self crossings neglected).

\section{Sausage growth with hydrodynamic friction.}

We shall focus our attention on the early stages where $L \gg r$. In this regime it is legitimate to ascribe most of the dissipation to hydrodynamic flows in the solvent near the sausage (at radial distances from the axis of order $r$ ). The contraction velocities are of order $\stackrel{\circ}{L}=\mathrm{d} L / \mathrm{d} t$, and the entropy source is

$$
T \stackrel{\circ}{S} \sim \eta\left[\frac{\circ}{r}\right]^{2} r^{2} L=\eta \stackrel{\circ}{L^{2}} L
$$

where $\eta$ is the solvent viscosity; $\stackrel{\circ}{L} / r$ is a typical velocity gradient, and $r^{2} L$ is the relevant volume. Various logarithmic factors associated with the flow field are omitted. The driving energy $F$ is the interfacial energy

$$
F=2 \pi L r \gamma=2 \Omega \gamma / r
$$

Remembering that $\Omega=\pi L r^{2}$ is conserved, we have

$$
\stackrel{\circ}{F}=\frac{-2 \Omega \gamma \stackrel{\circ}{r}}{r^{2}}=\frac{\Omega \gamma \stackrel{\circ}{L}}{L r} .
$$

Finally, writing $\stackrel{\circ}{F}=-T \stackrel{\circ}{S}$ we arrive at

$$
\begin{gathered}
L^{2} r \stackrel{\circ}{L}=-\gamma \Omega / \eta \\
{\left[\frac{L(t)}{L_{0}}\right]^{5 / 2} \cong 1-t / \tau_{\mathrm{c}}}
\end{gathered}
$$

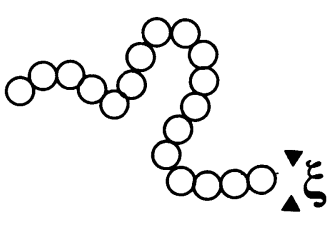

(a)

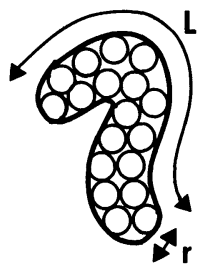

(b)

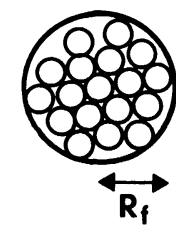

(c)

Fig. 1. - Successive stages of folding. (a) The original coil, represented in terms of subunits of size $\xi$. (b) The subunits tend to stick to each other, but do not overlap. The result is a "sausage ". (c) The final state is a compact sphere. A (possibly incorrect) feature of the model is the neglect of large loop conformation in stages (a) and (b). 
displaying a relaxation time

$$
\tau_{\mathrm{c}}=\frac{\eta L_{0}^{2}}{\gamma \xi}=\frac{\eta a^{3}}{k \theta} N^{2} \frac{\Delta T}{\theta} .
$$

The $N^{2}$ dependence of $\tau_{\mathrm{c}}$ is reminiscent of a Rouse relaxation time [12] but there is an added factor $\Delta T / \theta$. Note that $\tau_{\mathrm{c}}$ increases with $\Delta T:$ larger quench depths give slower relaxations.

An interesting presentation of this result has been suggested to us by a referee. This amounts to introduce the difference $\Delta T^{*}$ between the theta point and the critical point of a polymersolvent mixture

$$
\Delta T^{*} \cong \frac{\theta}{N^{1 / 2}}
$$

Then we have the scaling form

$$
\tau_{\mathrm{c}}=\tau_{\mathrm{z}}(\theta) \frac{\Delta T}{\Delta T^{*}}
$$

where $\tau_{\mathrm{z}}(\theta)\left(\sim N^{3 / 2}\right)$ is the Zimm relaxation time of the coil in ideal conditions.

Of course, the quench depth has a lower bound - quoted in section 1 . All our analysis assumed $N \gg g$ or $\Delta T / \theta>N^{-1 / 2}$. When we reach the limit $N=g$ (a single blob per chain) equation (7) reproduces the conventional Zimm relaxation time [13] of an ideal coil $\tau_{z}(\theta)$

$$
\tau_{\mathrm{c}}(g=N)=\eta a^{3} N^{3 / 2} / k \theta=\tau_{\mathrm{z}}(\theta) .
$$

Equation (6) describes most of the relaxation, and is applicable whenever $L \gg r$. There is a final period with $L \sim r$, where the sausage transforms into a sphere of radius

$$
R_{\mathrm{f}} \cong\left(\frac{N}{g}\right)^{1 / 3} \xi
$$

The relaxation in that final period is exponential, with a characteristic time

$$
\tau_{\mathrm{s}} \cong \frac{\eta R_{\mathrm{f}}}{\gamma}=\tau_{\mathrm{c}}\left(\frac{g}{N}\right)^{5 / 3} \ll \tau_{\mathrm{c}} .
$$

But, in this late stage, and for deep quenches ( $c_{g}$ large) reptation processes in the coil can dominate over solvent friction : the viscosity $\eta$ to be used in equation (10) may have to be redefined.

\section{Practical aspects}

3. 1 ORDERS OF MAGNITUDE. - The folding time $\tau_{\mathrm{c}}$ of equation (7) has a prefactor $\eta a^{3} / k \theta$ of order $10^{-12}$ seconds with low viscosity solvents. For a very long polystyrene chain $\left(M=10^{7}\right)$, taking $\Delta T / \theta=10^{-1}$ we arrive at time $\tau_{\mathrm{c}} \sim 1$ millisecond. In practice, one would probably prefer pressure jumps to temperature jumps, especially for lower molecular weights $M$. Experimentally, in the PS/cyclohexane system $(7,8)$, for $\Delta T / \Delta T^{*}>40$ and a molecular weight of $10^{7}$, precipitation occurs at concentrations $\widetilde{\sim} 10^{-5} \mathrm{~g} / \mathrm{cm}^{3}$.

3.2 Light SCATTERING. - If, by some trick we are able to register an intensity/angle curve (due to a very dilute suspension of chains) during intervals $\Delta t \ll \tau_{\mathrm{c}}$ we can monitor the radius of gyration $R_{\mathrm{G}}(t)$. Noting that the "sausage » is built around a statistical coil, we expect

$$
R_{\mathrm{G}}^{2}(t) \sim L r \sim(L \Omega)^{1 / 2}
$$




$$
R_{\mathrm{G}}^{2}(t)=\left(L / L_{0}\right)^{1 / 2} R_{\mathrm{G}}^{2}(0)
$$

where $L / L_{0}$ can be taken in equation (6).

3. 3 COMPUTER EXPERIMENTS. - It may be tempting to mimic this dynamical collapse by computer simulations. However, hydrodynamic backflows make this rather heavy. If we ignore backflows, we are led to a less physical, but simpler problem of the Rouse type. Equation (2) now reads

$$
T \stackrel{\circ}{S}=\zeta N \stackrel{\circ}{L}^{2}
$$

where $\zeta$ is a monomer friction coefficient. This leads to a collapse time $\tau_{\mathrm{c}}$ which is now exactly equal to the Rouse time of the original chain.

The problem without backflow may in fact be relevant for certain polypeptides where internal conformation barriers of the peptide chain are dominant. But we should repeat our words of caution : in any case, what is discussed here is an early stage of folding, for homopolymers in flexible conformations. All the intricacies related to the formation of ordered pieces $(\alpha, \beta, \ldots)$ are not included.

3.4 ENTANGLEMENT EFFECTS. - A long chain in $\theta$ conditions may be entangled [14]. There is no strong experimental evidence for this effect in dilute solutions. On the theoretical side, the number of self contacts of a $\theta$ chain at large scales is of order $N^{1 / 2}$. If a fraction $1 / N_{\mathrm{e}}$ of these contacts leads to entanglements we expect a significant change in the relaxation times whenever $N^{1 / 2} / N_{\mathrm{e}}>1$. If we (arbitrarily) insert for $N_{\mathrm{e}}$ the value which is adequate for a melt of polystyrene $\left(N_{\mathrm{e}} \sim 300\right)$ the critical value of $N$ is $\sim N_{\mathrm{e}}^{2} \sim 10^{5}$, corresponding to molecular weights $M \sim 10^{7}$. Thus, for most practical situations with PS samples, entanglements should not be dominant in the initial state, if our estimate of $N_{\mathrm{e}}$ makes sense.

\section{References}

[1] The folding protein problem, D. B. Wetlaufer ed., AAAS Selected symposium 89 (Westview, Boulder) 1984.

See also Dill, K. A., Biochemistry 24 (1985) 1501.

[2] De Gennes, P. G., J. Physique Lett. 44 (1983) L-225.

[3] See the review by Williams, C., Brochard, F., Frisch, H. L., Ann. Rev. Phys. Chem. 32 (1981) 443.

[4] Cuniberti, C., Bianchi, U., Polymer 15 (1974) 346.

[5] Slagowski, E., Tsai, B., McIntyre, D., Macromolecules 9 (1976) 687.

[6] Sun, S., Nishio, I., Swislow, G., Tanaka, T., J. Chem. Phys. 73 (1980) 5971.

[7] Persynski, R., Adam, M., Delsanti, M., J. Physique 45 (1984) 1765.

[8] Vidakovic, P., Rondelez, F., Macromolecules 17 (1984) 418.

Miyaki, Y., Fujita, H., Polymer J. 13 (1981) 749.

[9] Stockmayer, W., Macromol. Chem. 35 (1960) 54.

[10] Lifshitz, I. M., Grosberg, A. Y., Khokhlov, A. R., Rev. Mod. Phys. 50 (1978) 683.

[11] SANCHEZ, I., Macromolecules 12 (1978) 980.

[12] Rouse, P. E., J. Chem. Phys. 21 (1953) 1273.

[13] Ziмm, B., J. Chem. Phys. 24 (1956) 269.

[14] BrochaRD, F., De GenNes, P. G., Macromolécules 10 (1977) 1157.

Brochard, F., J. Physique 44 (1983) 39. 